\title{
Study on Replication Mechanism of Routines Based on Knowledge Perspective
}

\author{
Yanliang Chen, Junting Ye \\ Shandong Institute of Business and Technology \\ Yantai, China \\ chenyanliang412@sina.com
}

\begin{abstract}
Replication of routines is a kind of important value ceration strategy for organizations. However, the incompatibility between existing routines and new contexts in the process of applying existing routines to new contexts has become the core issue concerns organizations the most. Drawing on recent theories on organizational routines, the crossing macro gaps and researching on micro-level mechanism are found to be one of the useful keys to unlock the "block box" of replication process. On the basis of reinterpreting routines connotation with knowledge as the artifact, this paper regards knowledge template as a characterization of organizational routines' replication, and finally establishes the replication path.
\end{abstract}

Keywords-Replication of routines; Knowledge template; Replication path

\section{INTRODUCTION}

The evolutionary theory school, represented by Nelson and winter [1], clarifies the replication mechanism of organizational routines with genetic mechanism of "gene". They believe that applying existing routines to new organizational contexts would expand organizational boundaries, improve operational efficiency and elevate organizational competence, thus affirm that replication of routines is a kind of important value-creating strategy for organization. The research has been flourishing for a while, and the scope of research covers specific production technology, IT system, business template, retail chain store and franchise organization [2-6]. Most scholars launch around "knowledge transfer" when exploring replication of routines, and replication of routines is regarded as a management mechanism of flexible knowledge. Unfortunately, no matter is the life cycle extension [7], international development [8] or the best human resource practice unit transfer [9] replication of routines all remain at the macro level of knowledge transfer the micro-mechanism of the replication process, as a "black box" process, is ignored, and the lack of replication of routines micro-mechanism analysis is difficult to effectively solve the " replication dilemma ", which is described by Winter and Szulanski [10]: the gaming between standardization of organization and the competition of specific organizational context. In other words, how routines are beneficial to adjust and modify to apply existing routines in new contexts and match between existing routines and new contexts in the

*Sponsors: National Social Science Fund Project (15CGL027); National Natural Fund Project (71501113). process of replication of routines.

Based on the perspective of knowledge, this paper interprets the "black box" of replication process by breaking the routine entities to match the routine template and new environment. This study is of great significance, in theory, exploring the replication of routines can enrich and improve the innovation of relevant management transplantation theory, and it will be useful for compare management theory, crosscultural management, evolution of enterprise growth theory and other theory research. The analysis of knowledge transfer mechanism will enrich the theory of economic evolution, such as organizational knowledge innovation, evolution and related path dependence. From the perspective of management practice, clarifying replication of organizational routines is beneficial for Chinese enterprises through flexible competence to nurture more effectively new markets, and provide useful guidance, they also will help the existing market experience and template routine apply to the new context. For enterprises that are uneasy because of context turbulence, this research also provides a new idea and project to imitate, learn and innovate for Chinese and the west excellent business routines, and achieve everlasting development.

\section{REPLICATION OF ROUTINES WITH KNOWLEDGE REPRESENTATION: MICRO-LEVEL INTERPRETATION}

\section{A. Crossing gaps: from macro to micro}

The introduction of knowledge transfer greatly expands the understanding of the replication of organizational routines by evolutionary economics school, which enriches and perfects the theory system of organizational routines replication. However, too much attention to the macro- level analysis, which of the whole routines make routines replication into a rigid trap, unable to interpret and answer the adjustment and modification in the replication process, which is the core category of organizational routines replication mechanism. This is because, in a dynamic environment, if the routine is not fully understood correctly, the deviation that occurs may damage the whole routines replication activities, making it difficult to realize the purpose of the organizational expectations. Moreover, organizational routines have contextual dependency, and the characteristics and complexity of a particular environment (or context), such as an industry, 
can also have an important impact on the replication of routine The reason for this is that evolutionary economics, when it comes to researching replication of routine, usually chooses truncated, collective, recurring routine entities that do not explore the "fine particles" of routine connotations, ignore the inherent heterogeneity of routine. Therefore, routines are simply transferred from one organization to another with vague sets of knowledge, that is to say, the replication of routine only focus on the macro level of knowledge transfer, which obviously are not able to explain the management practice that is far more richer than the replication theory. Moreover, the existing research does not elaborate on the specific "mode" of the replication of routines. Instead, it simply takes the replication factor as the "sending-receiving", without further digging the form of replication factor transfer from the microscopic point of view or characteristics, nature can not clarify the specific replication mechanism of organizational routines.

In fact, organizational routines are not homogeneous entities. There are complex and diverse relationships between "fine particles", "components" and their mutual mapping. This is very important findings and basic premises of the study from a micro perspective on the organizational routines. This view does not lack theoretical basis. Nelson and Winter has found that organizational routines are hierarchical, and the connotation of low level routines and high-level routines are different. Feldman and Pentland believe that there are two forms of routines: ostensive and performative [11]. The former mainly involves abstraction level routines, such as tacit knowledge and rules, the latter mainly relates to the level of specific routines, in particular to have some specific action, a recursive relationship exists between the two factors; Pentland and Feldman developed behavioral and cognitive differences in routine through specific case described in detail to analysis how routine is established [12].This paper considers that opening replication black box process should start from the micro perspective, the theory of entity unconventional, indepth routine, starting from the routine of heterogeneity angle, according to routine different "particles and components" consciously or even two yuan to develop and deploy multiple thinking. The micro perspective, the heterogeneity of a natural problem is the organizational routines "fine" and "components" is very likely to be due to their respective connotation, nature and different mapping relationship, the evolution mechanism of differences exist in the process of selection and show different complex mechanism.

\section{B. Knowledge template and replication}

Knowledge becomes an interesting and meaningful concept when exploring the essence of routine and its replication from a microscopic level. This is because when knowledge is concerned with individual compatibility strategies, knowledge is the basis for all action strategies to involve another individual in making action strategies. Zollo and Winer believe that along this view, valuable knowledge is embedded in the routine[13], many scholars also believe that knowledge is the essence of routine, and replication as a core strategy is to "transfer", namely the routine from a sending unit to a receiving unit of the shift in the hope of receiving help units to create a "similar" routine, routine replication can be achieved through the knowledge transfer mechanism, this paper will define the knowledge transfer to send the source and receiver two yuan organization communication, that is to say replication depends on a basic hypothesis: the replication factor can be between source and receiver and flow the receiver can be put into practice, which involves in routine replication process of knowledge is in what form the flow of knowledge transfer and how to eliminate the obstacles in the process?

This study suggests that existing routines is not a unit knowledge structure but an aggregation of knowledge, that is to say the routine is made up of a number of different unit knowledge structures (or called meta-knowledge) compile a combination of knowledge based on certain principles, after repeated strengthen times, or when these aggregations of knowledge with inertia (characteristics of the routine), they will solidify into a series of different "template", thus becoming the core of organizational routines. More importantly, a large number of studies confirm that template is regarded as an important way to overcome the knowledge stickiness of embedded routines and eliminate knowledge transfer barriers, strictly enforcing the template will increase the performance of receiving replication cells, moreover, as time goes on, the recipient will "control" the organizational routines of replication, which enhance their adaptability and provide valuable feedback to the templates of routines; The study of Jensen and Szulanski [14] show that templates reduce the obstacles and resistance of recipients to accept new knowledge; Meanwhile, it also shows that the ability and willingness of the recipient are also important factors and conditions that affect the replication of the template, such as the recipient's knowledge background, absorptive capacity, and the organization's trust mechanism and so on. The knowledge attribute of routines indicates that the knowledge structure of these templates is not exactly the same and the same monomer knowledge will show different characteristics due to different composite structures, the diversity and differences of routines. It can be inferred that these templates of routines that compiled based on certain sequence structure combination are replicating, which is not the transfer of knowledge in general, but depends on the participation and efforts of the agent of routines. Specifically, the members of the organization need to deconstruct the template, distinguish the different unit knowledge contained in the template, and clarify the knowledge combination sequence structure of the template. 


\section{REPLICATION MECHANISM OF ROUTINES}

\section{A. Routine hierarchy and template classification:the division} of this paper

Organizational routine is not a vague, indivisible and ambiguous entity, its interior has a lot of fine particles and components of different properties, characteristics and mechanism of action. The introduction of knowledge templates provides a visual and operable form for in-depth analysis the "black box" of routines replication process. Therefore, it is not difficult to understand that these different fine particles and components may appear different form of template after the "knowledge structure-reductive combination".

The division of routines from the ability dimension helps to explore how enterprises improve their adaptability through the development and deployment of routines, and context dependence of routines in the face of a complex and changeable external context, while it is more helpful to explain the mechanism of routines in the management practice. Therefore, this study will follow this dimension and distinguish regular routines from flexible routines combined with specific research needs, the former mainly refers to the general operational ability of the organization, while the latter refers to the dynamic ability of the organization. From view of knowledge connotation, the nature and meaning of the two types of routines are different, the knowledge embedded in the regular routine template should be relatively clear and specific, the connotation of each unit knowledge is clearer, while the knowledge combination structure is relatively stable, and the evolution of knowledge is generally shown as continuity and asymptotic behavior. From view of routines context dependency, it is possible to speculate that such routines usually show higher levels of replication when the new context is more stable and less competitive; the flexible routine is different, the embedded knowledge is more diverse and some tacit knowledge connotation may not clear, while the composite structure of knowledge is complicated and the evolution of knowledge is not continuous and radical, so the kind of routines replication can show higher levels of replication in unstable context and weak competition.

\section{B. Context dependency of replication}

The routines' replication has context dependency, that is, the matching of the relationship between routines and environment in the process of replication stems from the interaction between routines and external context, the routines' replication in different environments shows different effectiveness. Regular routines are more suitable for replication in a more stable environment, while flexible routines are more likely to interact well with dynamic environments, so the four matching types can be divided into two groups. Scaffolded Action and Situated Action illustrate how behavior depends on external support, and external structures help to control, instigate and coordinate individual behavior. And different context can lead to situations that the general rules are applied to specific scenarios where there may be insufficient detail and loss, that is, the effectiveness of replication may be different, and contextual relationship lead to a great deal of difference in the ability to replicate, the degree of replication, and the adaptation ability in the later period. The most important manifestation of the specificity of routines is that the transfer to other situations will be restricted, and when they are removed from their original scenarios, they may become meaningless and their "productive" capacity may decline. As a result, table 1 is formulated.

TABLE I. REPLICATION AND DIFFERENT ENVIRONMENTS

\begin{tabular}{|c|c|c|c|c|}
\hline Environment & Template of routines & Effect of replication & Efficiency of replication & Results \\
\hline Stable & Regular routines & High & High & Sensitivity \\
& Flexible routines & High & Low & Insensitivity \\
\hline Turbulent & Regular routines & Low & Low & Insensitivity \\
& Flexible routines & High & High & Sensitivity \\
\hline
\end{tabular}

\section{Replication path of organizational routines}

The core topic of "replication dilemma" is that when the organization "applies" the routines to different environments, the routines template will not be able to make useful adjustments and modifications because of the different contexts. In this paper, the "application" of routines template (that is routines' replication) needs more organization under the premise of profound understanding of routines template, through existing knowledge and learning ability, perception, recognition, classification, and organization replication. Expand organizational boundaries, promote business processes, improve organizational efficiency, pursue shortterm benefits, obtain the path dependency of positive feedback and profit to achieve organizational optimization. It can be seen that the replication of regular routines are much more matching with stable environments. However, in the turbulent or new environment, the "application" of flexible routines template needs more organization to understand and interpret the routines template more deeply. Search, adjust, and experiment by consciously creating, expanding, and improving the knowledge and learning capabilities of organization. Through organizational replication, enhance organizational adaptability, improve organizational learning ability, pay attention to long-term development, strengthen the ability of organizational integration and allocation. Therefore, the replication of flexible routines are much more matching with the new and turbulent environments.

When the environment is relatively stable, the replication of regular routines template is easy to be favored by organizations. Of course, this paper does not deny the possibility of flexible routines replication in this environment. But as mentioned above, because the knowledge connotation 
of flexible routines is not clear, the composition structure is complex and changeable, and the requirement of the organizational ability is high. Considering the principle of "satisfaction" in management and the scarcity of resources, organizations usually choose the template of regular routine first, and the connotation structure of this kind of routine is relatively stable and shows a certain degree of asymptotic, which is more consistent with the external stable environment, and the organization will give priority to match it. The replication of regular routines template depends on the exploitation competence of the organization. The relationship between them is type-sensitive and situational matching, and the replication effect and efficiency are both higher at this time, which means that the organization can achieve the desired purposes of routine replication, so this matching will be adopted by the organization. However, the exploration competence can also achieve better effect of replication, but the efficiency of replication is lower, that is to say, the "cost" of replication will be higher, so it will not be adopted by the organization; When the organization is in a turbulent environment with high competitive pressure, because of its clear connotation and stable structure, the regular routines template is difficult to adapt to the dynamic environment, so the flexible routines is more suitable for the organization to replicate. This kind of replication depends on the exploration competence of the organization, the relationship between them is type-sensitive and situational matching. At this time, the effect of replication and the efficiency of replication are both higher, which means that the organization can achieve the expected goal of routine replication. Therefore, this matching will be adopted by the organization, and although the exploitation competence can accomplish the routines' replication efficiently, the quality of the replication is not high, and it is difficult to adapt to the rapidly changing external environment. It can cause replication to fail by plunging your organization into Core Rigidity and Competence Trap, and will not be adopted by the organization.

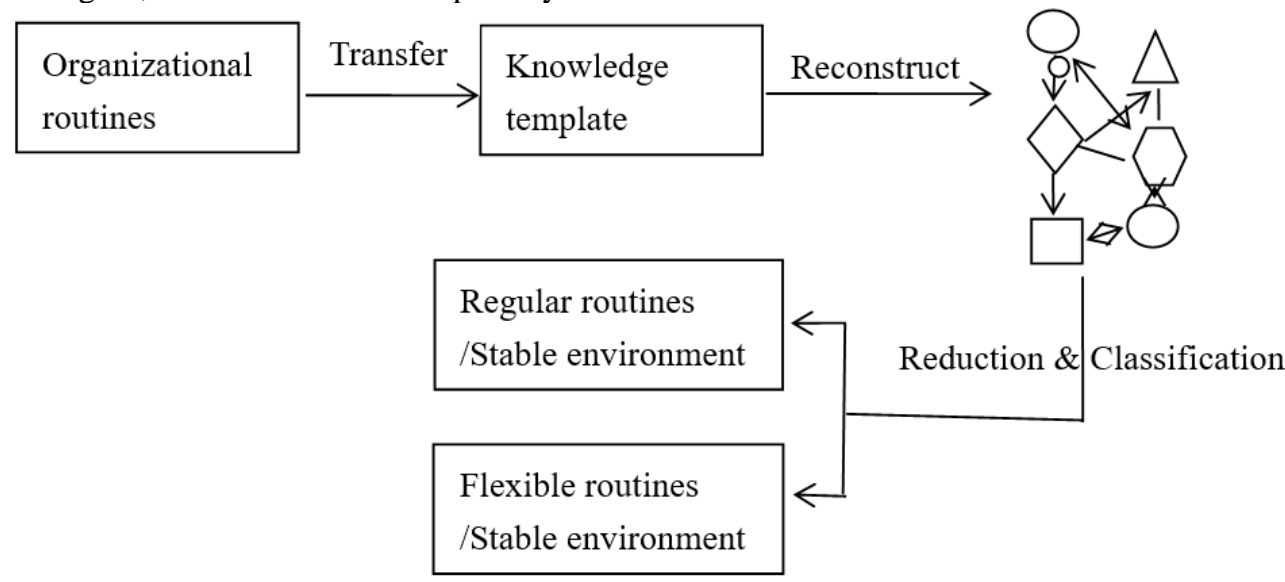

Fig. 1. Replication Path of Organizational Routines

\section{CONCLUSION}

As an important way to achieve internationalization, replication of routines is getting extensive attention of academic circles. The paradigm of traditional research follows the evolution economics, and keep the macro analysis of the replication, the discretion of management does not get more attention and respect, the effort and participation of individual agency and members are consciously or unconsciously ignored, so, it is difficult to solve the riddle of replication and provide substantial guidance to enterprise. This study abandon the expression of traditional evolutionary economics to "routines", which is considered "habits, genes and models", explores transfer mechanism of replication to break the "black box" on the micro level. The study also receives the following three important conclusions: (1) routine is not a whole entity, from the knowledge perspective combined with heterogeneity and level of routine, routine can be divided into normal routine and flexible routine, in development, the two types of routines are expounded on the connotation and important characteristics. (2) The study achieves matching of different routines and organizational situation based on the situation theory. (3) Finally, it establishes the replication path based on the analysis.

\section{REFERENCES}

[1] Nelson, R. R., S. G. Winter. An Evolutionary Theory of Economic Change [M]. Harvard University Press, 1982.

[2] Ruuska, I., T. Brady. Implementing The Replication Strategy in Uncertain and Complex Investment Projects [J]. International Journal of Project Management, 2011, 29(4): 422-431.

[3] D'Adderio, L. Configuring Software, Reconfiguring Memories: The Influence of Integrated Systems on The Reproduction of Knowledge and Routines [J]. Industrial and Corporate Change, 2003, 12(2): 321 350 .

[4] Aspara, J., J. Hietanen., and H. Tikkanen. Business Model Innovation vs Replication: Financial Performance Implications of Strategic Emphases [J]. Journal of Strategic Marketing, 2010, 18(1): 39-56.

[5] Yaniv, E., D. M. Brock. Replication Strategies, Knowledge and Attention: A Study of Coffee Chains [J]. International Journal of Service Industry Management, 2008, 19(3):379-399.

[6] Szulanski, G., R. J. Jensen. Growing Through Copying: The Negative Consequences of Innovation on Franchise Network Growth [J]. Research Policy, 2008, 19(3): 379-399.

[7] Helfat, C. E., M. A. Peteraf. The Dynamic Resource-based View: Capability Lifecycles [J]. Strategic Management Journal, 2003, 24(10): 997-1010. 
[8] Jonsson, A., N. J. Foss. International Expansion Through Flexible Replication: Learning From The Internationalization Experience of IKEA [J]. Journal of International Business Studies, 2011, 42(9): 1079-1102.

[9] Lervik, J. E., B. W. Hennestad, and R. P, Amdam et al. Implementing Human Resource Development Best Practices: Replication or Re-creation? [J]. Human Resource Development International, 2005, 8(3): 345-360.

[10] Winter, S. G., G. Szulanski. Replication as Strategy [J]. Organization Science, 2001, 12(6): 730-743.

[11] Feldman, M. S., B. T. Pentland. Reconceptualizing Organizational Routines as A Source of Flexibility And Change [J]. Administrative Science Quarterly, 2003, 48(1):94-118

[12] Pentland, B. T., M. S. Feldman. Designing Routines: on The Folly of Designing Artifacts, While Hoping for Patterns of Action [J]. Information and Organization, 2008, 18(4):235-250.

[13] Zollo, M., S. G. Winter. Deliberate Learning And The Evolution of Dynamic Capabilities [J]. Organization Science, 2002, 13(3): 339-351.

[14] Jensen, R. J., G. Szulanski. Template Use and The Effectiveness of Knowledge Transfer [J]. Management Science, 2007, 53(11): 17161730 . 